\title{
Increasing antibiotic resistance among uropathogens isolated during years 2006-2009: Impact on the empirical management
}

\author{
Hamid Mohammad-Jafari, Mohammed Jafar Saffar, Ibrahim Nemate, Hana Saffar, Ali-Reza Khalilian
}

Department of Pediatric Nephrology (HMJ) Department of Pediatric infectious diseases (MJS), Department of Clinical Microbiology (IBN), Department of Biostatics (ARK) Mazandaran University of Medical Sciences, Sari and Department of Pathology (HS), Tehran University of Medical Sciences, Tehran, Iran

\section{ABSTRACT}

Urinary tract infections (UTI) are one of the most common infections with an increasing resistance to antimicrobial agents.

Purpose: Empirical initial antibiotic treatment of UTI must rely on susceptible data from local studies.

Materials and Methods: Retrospective analysis of isolated bacteria from children with UTIs was performed at the university hospital during years 2006-2009. The findings were compared with data collected in a similar study carried out in 2002- 2003.

Results: A total of 1439 uropathogens were isolated. Escherichia coli (E.coli) was the leading cause, followed by Enterobacter, and other gram negative bacilli. It was observed resistance of E.coli to ceftriaxone, cefexime, amikacin, gentamycin, and nalidixic acid; Enterobacter to cefexime; and the resistance of gram negative bacilli to gentamicin and cefexime increased significantly. The highest effective antibiotic was Imipenem, ciprofloxacin, and amikacin with 96.7\%, 95\% and 91\% sensitivity rates, respectively, followed by ceftriaxone $77.2 \%$, gentamicin $77 \%$, nitrofurantoin 76.4\%, nalidixic acid 74.3\% and cefexime with 70\%.

Conclusion: The use of nitrofurantoin or nalidixic acid as initial empirical antibacterial therapy for cystitis seems appropriate. For cases of simple febrile UTI, the use of initial parenteral therapies with amikacin or ceftriaxone followed by an oral third generation cephalosporin also seemed appropriated, and in cases of severely ill patients or complicated UTI, imipenem as monotherapy or, a combination of Ceftriaxone with an aminoglycoside, are recommended.

\section{ARTICLE INFO}

\section{Key words:}

Urinary tract infections;

children; antibacterial agents

Int Braz J Urol. 2012; 38: 25-32

Submitted for publication:

October 27, 2010

Accepted after revision:

June 22, 2011

\section{INTRODUCTION}

Urinary tract infection (UTIs) are common in childhood, occurring in about 5\% of febrile infant and $2 \%$ of febrile children $<5$ years of age ( 1 3). UTIs pose a significant health risk due to progress to urosepsis, renal scarring and/or progressive kidney damage (4-6). The prognosis depends on timely administration of appropriate antimicrobial treatment. Antibiotic therapy is generally initiated immediately and empirically even before bacteriological culture results are available (1-6). There is a growing concern about the increasing antimicrobial resistance of the causative organisms of UTIs worldwide (7). The increasing resistance trends are likely to have important clinical 
implications for the empirical use of antibiotics $(8,9)$. When prescribing empirical therapy for infections in the community and in the hospital, it is important to know the national and local antibiotic resistance patterns of different organisms. This requires permanent monitoring to gatter information for providing new therapeutic options (7-9). The aim of this study was to determine the relative frequency of bacterial species causing UTI and their susceptibility patterns to most commonly used antibiotics during a four years-study period (2006-2009). Also, in order to document the resistance trends of isolated uropathogens to these drugs, the collected data were compared to the findings of a similar earlier report (10) to evaluate the most appropriate options for initial empirical therapy of UTI for children in the region.

\section{MATERIALS AND METHODS}

This retrospective study analized the bacteria isolated from patients with UTI in a teaching hospital at the main center for newborns located in Sari, capital of Mazandaran Province, North of Iran. Urine samples were obtained from ambulatorial and in-patients with suspected UTI and who were admitted in pediatric wards with signs and symptoms of UTI, to document the common bacterial species causing UTI, and their antibacterial susceptibility profile. The period of analysis was 2006 to 2009. Collected data were also compared to findings of a similar earlier study (10) (at the same hospital with similar methods and including criteria) to determine the resistance trends of isolated uropathogens. Urine samples were obtained before the use of antimicrobial agents by bladder aspiration, urethral catheterization or clean-catch midstream methods.

Patients were children aged from 1 week to 12 years without history of genitourinary abnormalities, recent hospitalization or antibiotic usage. Patients were hospitalized for evaluation and treatment in pediatric-neonatal wards with signs and symptoms of acute pyelonephritis or cystitis, including: temperature $\geq 38^{\circ} \mathrm{C}$, chills, frequency, dysuria, urgency, suprapubic and/or flank tenderness, pyuria (defined as $\geq 5 \mathrm{WBC} / \mathrm{Hpf}$ ), fever with unknown source in children, and in neonates
(7-30 days of age) with clinical evidences of sepsis [community-acquired UTI (CA-UTI)]. Quantitative urine cultures were performed on a blood agar base plate [Merck ${ }^{\circledR}$, Germany] and eosin methylenblue agar plate [ATD, Antec and Diagnostic $\left.{ }^{\circledR}, \mathrm{UK}\right]$. The plates were incubated at $37^{\circ} \mathrm{C}$ for 24 hours. A positive urine culture was defined as the growth of $\geq 105, \geq 104$, and any colony-forming unit [CFU]/ $\mathrm{mL}$ of a single uropathogen for specimens obtained by clean-catch midstream, catheterization, and suprapubic methods, respectively. The bacteria were identified by conventional procedures. Antibiotic sensitivity testing was performed using the Kirby-Bauer Disk diffusion method [Padtan$\mathrm{Teb}^{\circledR}$, Tehran] according to the recommendations of the National Committee for Clinical Laboratory Standards. Antibiotics tested for gram-negative uropathogens were: ceftriaxone [CTX], amikacin [AMK], gentamycin [GEN], ampicilin [AMP], cotrimoxazole [C-tri], ciprofloxacin [CIP], nitrofurantoin [NF], nalidixic acid [NA], imipenem [IMP] and cefixime [CFX]. In all uropathogens, the yearly susceptibility profiles and resistance trends to each antibiotic was calculated and compared to the results of an earlier study (2002-2003 period) using descriptive statistical methods. The relative role of uropathogens causing UTI and their susceptibility levels to an antibiotic were determined by dividing the total numbers of susceptible organisms to the total numbers of uropathogens isolated during the 4-year study period. The trend 2-test and Fisher exact probability test were used for statistical comparison between the 4 years of this period of study and with the study of 2002-2003 for each uropathogen and each antibiotic. $\mathrm{P}$ value $<0.05$ was considered significant.

\section{RESULTS}

From January 2006 to 2009, a total of 12983 urine samples were submitted for analysis and culture. These samples showed 1439 bacterial isolates. Enteric gram-negative bacilli accounted for 1419 of 1439 (98.6\%) of isolates. Escherichia coli (E.coli) was the main uropathogen (865 (60\%) of isolates) followed by: Enterobacter 248 (17.2\%), and other gram negative bacilli (GNB) including Proteus, Klebsiella, Pseudomonas etc 
(306 (21.2\%)). In the initial study (January 2002 to 2003) 404 uropathogens from 3363 urine samples were isolated. The results are shown in Table-1.
(Table-2). When these findings were compared with similar data collected during 2002-2003, the results indicated an statistically significant in-

Table 1 - Relative distribution of submitted urine samples and isolation rates of bacterial species causing urinary tract infections based on the year of study, Sari-Iran.

\begin{tabular}{lcccccc}
\hline Study year & 2006 & 2007 & 2008 & 2009 & Total & 2003 \\
\hline No of submitted samples & 2156 & 2210 & 4219 & 4398 & 12983 & 3363 \\
& 220 & 226 & 472 & 521 & 1439 & 404 \\
No of positive(\%) samples & $(10.2)$ & $(10.2)$ & $(11)$ & $(12)$ & $(11)$ & $(12.5)$ \\
& 132 & 140 & 282 & 311 & 685 & 290 \\
E.coli (\%) & $(60)$ & $(62)$ & $(59.7)$ & $(59.7)$ & $(60)$ & $(72)$ \\
& 42 & 36 & 78 & $92(17.6)$ & 248 & 23 \\
Enterobacter(\%) & $(19)$ & $(16)$ & $(16.5)$ & & $(17.2)$ & $(5.7)$ \\
& 46 & 50 & 101 & $109(21)$ & 306 & 94 \\
Gram negative bacilli(\%)* & $(21)$ & $(22)$ & $(21.4)$ & & $(21.2)$ & $(15.8)$ \\
Others & - & - & 11 & $9(1.7)$ & - & 27 \\
& & & $(2.3)$ & & & $(6.7)$ \\
\hline
\end{tabular}

${ }^{*}$ Gram negative bacilli mainly included Proteus, Klebsiella and Pseudomonas

The antimicrobial susceptibility profiles and resistance trends of different isolated uropathogens during the study period (2006-2009) are shown in Figure-1. Overall, the results revealed that there was a statistically significant increase of the resistance prevalence of isolated pathogens to some tested antibiotics from 2006 to 2009. However, this trend was more prominent for E.Coli than to other uropathogens.

During the study period (2006-2009) E.coli resistance prevalence to CTX, CFX, AMK, GEN and NA increased significantly (Figure-1). For Enterobacter this trend was observed only for CFX and for other gram negative bacilli to CFX and GEN (Figure-1). Based on the results of this study, the first three most effective antibiotics for initial empiric therapy were IMP, CIP, and AMK with $96.7,95$, and 91\% susceptibility rates respectively. These were followed by CTX (77.2\%), GEN (77\%) NF (76.4\%), NA (74.3\%), and CFX (70\%) crease prevalence of resistance of E.Coli to most comparable data except AMP and C-tri, which showed a significant decrease (Figure-2). For other uropathogens, this pattern was not observed and the susceptibility rates for most tested antibacterial agents did not change significantly from the years 2002-2003 to 2006-2009. However, due to the decreasing relative role of infection with Pseudomonas spp during years 2006-2009 (data not shown), the sensitivity of gram negative bacilli to CTX improved significantly as compared to the year 2003.

\section{DISCUSSION}

As expected and demonstrated by earlier reports $(1-3,10)$, E.coli was the main pathogen causing UTI among our community-acquired or hospital treated patients, followed by other enteric gram negative bacilli (GNB). Decreased rela- 
Figure 1 - The susceptibility percentile of uropathogens isolated during years 2006-09 to each antibiotic, Sari-Iran.
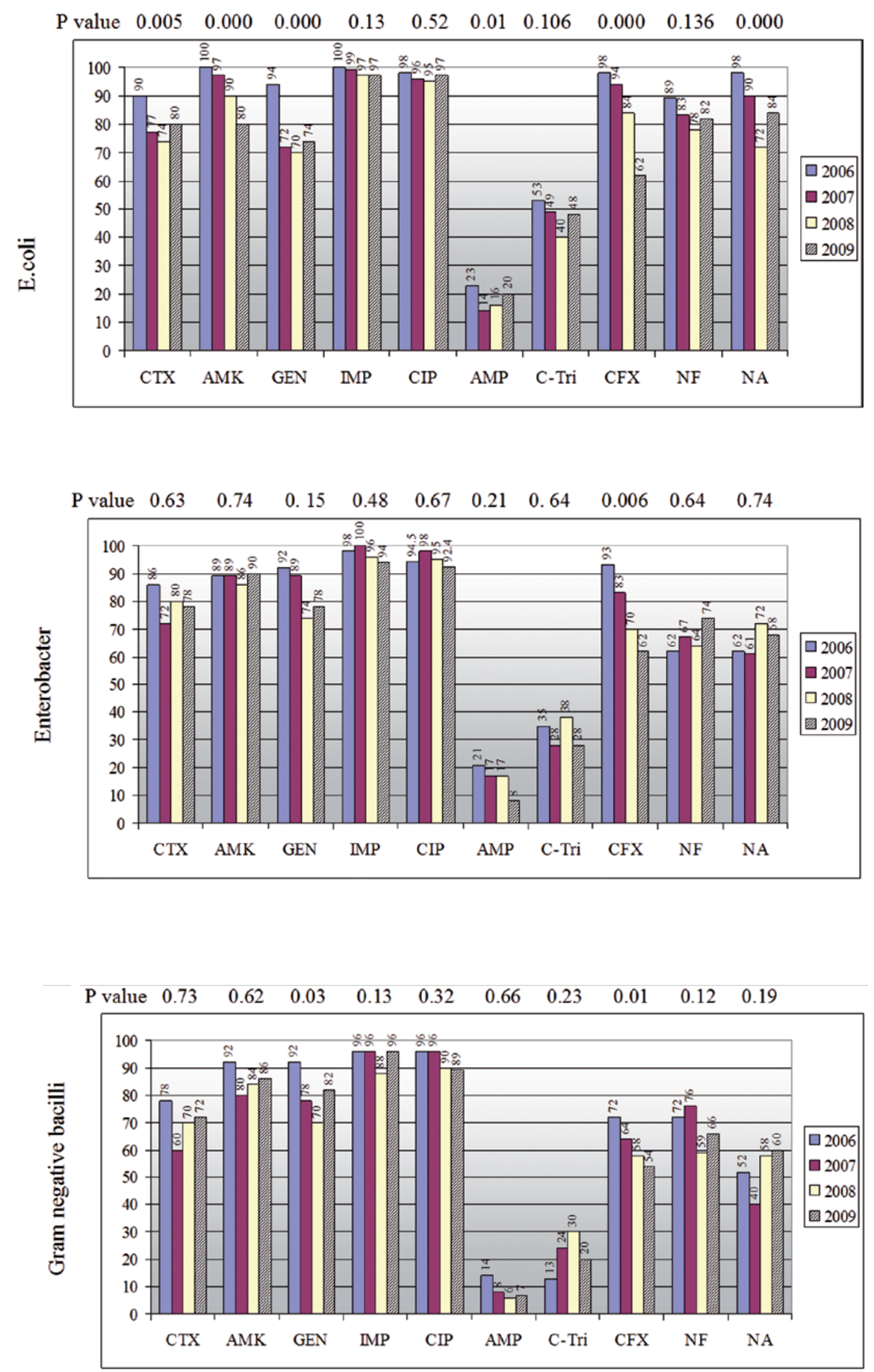
Table 2 - Relative chance of susceptibility for patients with UTI based on the sensitivity levels of 1419 uropathogens isolated during 2006-2009.

\begin{tabular}{|c|c|c|}
\hline Antibiotics & No of susceptible isolated pathogens (total of 1419) & $\%$ Susceptibility \\
\hline $\mathrm{CTX}^{\mathrm{b}}$ & 1095 & 77.2 \\
\hline AMK & 1288 & 91 \\
\hline GEN & 1092 & 77 \\
\hline IMP & 1372 & 96.7 \\
\hline CIP & 1346 & 95 \\
\hline AMP & 176 & 12.5 \\
\hline C-Tri & 563 & 40 \\
\hline CFX & 994 & 70 \\
\hline NF & 1083 & 76.4 \\
\hline NA & 1054 & 74.3 \\
\hline
\end{tabular}

a: the percentage denotes the mean average susceptibility levels for a 4- year period of study to each agent.

b: CTX: ceftriaxone, AMK: amikacin, GEN: gentamycin, IMP: imipenem, CIP: ciprofloxacin, AMP: ampicillin, C-tri: co-trimoxazole, CFX: cefexime, NF: nitrofurantin, NA: nalidixic acid.

Figure 2 - Comparison between average susceptibility level of E.coli isolated during years 2006-09 with those of 2003. Sensitivity levels to AMP and C-Tri decreased significantly from the years 2003 to 2006-2009.

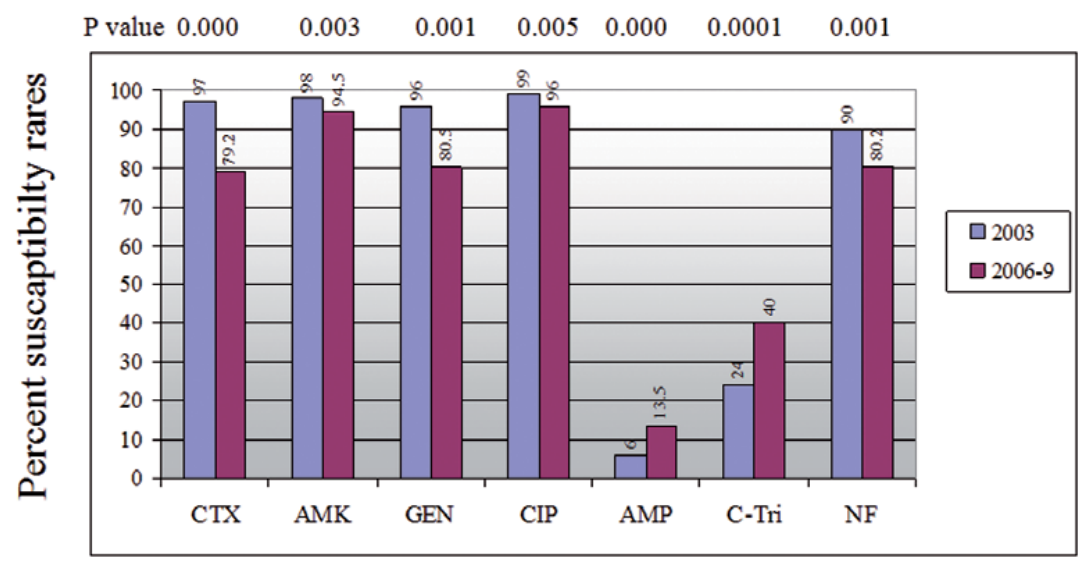


tive prevalence of E.coli from 72\% to 60\% and increased rate of Enterobacter and other GNB from the year 2003 to 2006-2009 are significant. The real causes for this difference despite similarity between sample sources and methods of the two studies are not known. However, depending of some characteristics and settings, as gender, younger age, previous antibiotic use or antibacterial prophylaxis, history of UTI, and urinary system anomalies, the role of E.Coli causing UTI may be lower (11).

When the susceptibility levels of uropathogens isolated during this study period to each antimicrobial agents were compared with similar data collected during 2002-2003 (10), it was observed a significant decrease of the sensitivity levels of E.coli to most tested antibiotics except AMP and C-tri, which increased (Figure-2). However, this pattern was not observed for other uropathogens. Despite decreased resistance levels of uropathogens to AMP and C-tri from year 2003 to 2009, the mean risk of resistance to both agents during 2006-2009 were 87.5\% and 60\% respectively (Table-2). Therefore, these drugs as a single agent for empiric therapy of patients suspected with UTI are not appropriate.

During recent years, the third cephalosporins and aminoglycosides are the preferred choice for initial empirical treatment of febrile children suspected with UTI $(12,13)$. In this study, the susceptibility prevalence of E.coli as of most common uropathogens decreased significantly to the tested third cephalosporins and aminoglycosides; P 0.005, 0.000, 0.000, and 0.000 to CTX, CFX, AMK, and GEN respectively. For other uropathogens, these patterns of resistance were not observed except for CFX to Enterobacter and also for GNB to GEN (Figure-1). Other studies in Iran have also revealed a similar resistance rate of E.coli isolated from out-and-in children patients with UTI; 25\%, 18.1\%, and 23.5\% in Tehran (14), and 21.5\%, 9.9\%, and 22.4\% in Ghazvin (15) to CTX, AMK, and GEN respectively. However, lower resistance rates were reported in some developing $(16,17)$ and developed countries $(8,11,18,19)$. These patterns of susceptibility put a patient with UTI at 22.8\%, 30\%, 9\% and 23\% risk of resistance to CTX, CFX, AMK, and GEN, respectively (Table-2). Therefore, for young infants, toxic patients or those with complicated UTI, CTX, CFX, or GEN monotherapy seems to be inadequate for most uropathogens causing UTI.

There is much evidence suggesting a relationship between prescribing habits and antibacterial resistance (20-23). As in many countries, in Iran and in our region, newer cephalosporins are the favorite antimicrobial agents for empirical treatment of not only UTI but also community-acquired respiratory and gastrointestinal infections $(24,25)$. Moreover, in secondary and tertiary care centers, newer cephalosporins and aminoglycosides are widely used. These high levels of resistance demonstrated for these drugs may be explained by high and uncontrolled use of these antibiotics in Iran and in our region. These drugs are prescribed not only for patients suspected with UTI but also for infection in other body sites or any febrile illnesses by physicians and even are used by the patient or family without prescription $(24,25)$. A study determined the rate of antibiotic prescription among outpatients referred to physicians in the community and showed that $\geq 50 \%$ of all patients and $\geq 79 \%$ of children received at least one systemic antibacterial agent (24).

Flouroquinolones are not the first-line drugs for initial treatment of UTIs and/or other infections in children. In this study, CIP was used to determine the susceptibility profiles of isolated uropathogens to this agent. Results indicated that most isolated pathogens were highly susceptible to CIP. Therefore, this drug may be considered a reserved alternative for children with difficult-totreat UTI. However, it is of concern that E.coli resistance to CIP increased significantly from years 2002-2003 to 2006-2009 (Figure-2). There was not a significant increase of resistance of isolated uropathogens to IMP over the study period (Figure-1). The susceptibility levels to IMP was higher and appropriated and was the most effective antibacterial agent tested in this study (mean susceptibility level 96.7\%) (Table-2). These levels of efficacy make this antibiotic a suitable choice for initial empirical treatment of cases with toxicity or difficult-totreat UTI (26). The most probable cause for these low resistance levels to CIP and IMP may be due to restricted use of these drugs in the community and/or the hospital. 
NA as well as NF, two oral drugs that are not systemic antibacterial agents, are widely used to treat cases with uncomplicated cystitis (afebrile UTI). Both drugs usually achieve excellent urinary concentration and exceeding MIC for the respective uropathogen. In vitro resistance does not necessarily mean unsuccessful therapy (27). During the 4-years study period susceptibility profiles of uropathogens to both agents did not changed significantly except E.coli to NA: P $=0.000$. Overall, mean susceptibility levels of uropathogens to NF and NA were $76.4 \%$ and $74.3 \%$ respectively. These levels of susceptibility make these antibiotics an appropriate option for initial empirical therapy of older children with cystitis. As a rule, these agents should not be used for febrile UTI.

It is quite alarming to note that almost all of the E.coli isolated in this study were found to become more resistant to most routine antibiotics that were tested. Therefore, this is an important issue to be addressed by the policy makers to formulate a strict antibiotic prescription policy in our country and region. Because the patterns of sensitivity of microorganisms to antibiotics vary over time and among different geographical location, empiric antibacterial therapy of infections should be based on a local experience of susceptibility and resistance profile.

\section{CONCLUSIONS}

Based on the results of this study, for children with simple cystitis, NF or NA monotherapy may be appropriate. In patients with simple febrile UTI, AMK or a short course of parenteral followed by oral therapy with a third generation cephalosporin seems reasonable. However, in cases with complicated UTI (young infants, severity ill and toxic patients, with underlying genitourinary anomalies, long-term use of urinary catheter or antibiotic prophylaxis, and nosocomial UTI) parenteral IMP monotherapy or a combination of a third generation cephalosporin with an aminoglycoside is recommended.

\section{CONFLICT OF INTEREST}

None declared.

\section{ACKNOWLEDGEMENT}

This project was funded by Vice-chancellor for Research Mazandaran Medical University. Special thanks to Salehifar PhD for helping editing the manuscript.

\section{REFERENCES}

1. Hoberman A, Chao HP, Keller DM, Hickey R, Davis HW, Ellis $D$ : Prevalence of urinary tract infection in febrile infants. $J$ Pediatr. 1993; 123: 17-23.

2. Schlager TA: Urinary tract infections in infants and children. Infect Dis Clin North Am. 2003; 17: 353-65.

3. Santen SA, Altieri MF: Pediatric urinary tract infection. Emerg Med Clin North Am. 2001; 19: 675-90.

4. Foxman B: Epidemiology of urinary tract infections: incidence, morbidity, and economic costs. Am J Med. 2002 113(Suppl 1A): 5S-13S.

5. Winberg J, Bollgren I, Källenius G, Möllby R, Svenson SB: Clinical pyelonephritis and focal renal scarring. A selected review of pathogenesis, prevention, and prognosis. Pediatr Clin North Am. 1982; 29: 801-14.

6. Jakobsson B, Berg U, Svensson L: Renal scarring after acute pyelonephritis. Arch Dis Child. 1994; 70: 111-5.

7. Gupta K: Emerging antibiotic resistance in urinary tract pathogens. Infect Dis Clin North Am. 2003; 17: 243-59.

8. Haller M, Brandis M, Berner R: Antibiotic resistance of urinary tract pathogens and rationale for empirical intravenous therapy. Pediatr Nephrol. 2004; 19: 982-6.

9. Sefton AM: The impact of resistance on the management of urinary tract infections. Int J Antimicrob Agents. 2000; 16: 489-91.

10. Saffar MJ, Enayti AA, Abdolla IA, Razai MS, Saffar H: Antibacterial susceptibility of uropathogens in 3 hospitals, Sari, Islamic Republic of Iran, 2002-2003. East Mediterr Health J. 2008; 14: 556-63

11. Lutter SA, Currie ML, Mitz LB, Greenbaum LA: Antibiotic resistance patterns in children hospitalized for urinary tract infections. Arch Pediatr Adolesc Med. 2005; 159: 924-8.

12. Elder GS: Urinary tract infection. In: Kliegman RM, Behrman RE, Jenson HB, Stanton BF. Nelsons Textbook of Pediatric. 18th ed. Philadelphia, Pa: Saunders Elsevier, 2007; pp 2223-8.

13. Hodson EM, Willis NS, Craig JC: Antibiotics for acute pyelonephritis in children. Cochrane Database Syst Rev. 2007; 4 : CD003772.

14. Afsharpayman Sh: Etiology and antibacterial resistance of isolated urophatogen in children in Najmiah Hospital, Tehran Iran. Fifth annual congress of Iranian Society of Pediatric Infectious Diseases and the first memorial of prof Ahmad Siaddati, 26-27 Nov.2010; Tehran Iran. pp. 145-52. 
15. Aiazi $P$, Jahani-Hashami $H$, Khabiri S: Etiology and antibacterial resistance patterns of urinary tract infections in children in Ghods Hospital Ghazvin, Iran. Third annual congress of Iranian Society of Pediatric Infectious Diseases. 23-24 Apr 2008; Tehran-Iran, pp. 32-6.

16. Yüksel S, Oztürk B, Kavaz A, Ozçakar ZB, Acar B, Güriz H, et al. Antibiotic resistance of urinary tract pathogens and evaluation of empirical treatment in Turkish children with urinary tract infections. Int J Antimicrob Agents. 2006; 28: 413-6.

17. Kurutepe S, Surucuoglu S, Sezgin C, Gazi H, Gulay M, Ozbakkaloglu $B$ : Increasing antimicrobial resistance in Escherichia coli isolates from community-acquired urinary tract infections during 1998-2003 in Manisa, Turkey. Jpn J Infect Dis. 2005; 58: 159-61.

18. Bean DC, Krahe D, Wareham DW: Antimicrobial resistance in community and nosocomial Escherichia coli urinary tract isolates, London 2005-2006. Ann Clin Microbiol Antimicrob. 2008; 18: 7-13.

19. Abelson Storby K, Osterlund A, Kahlmeter G: Antimicrobial resistance in Escherichia coli in urine samples from children and adults: a 12 year analysis. Acta Paediatr. 2004; 93: 487-91.

20. Bronzwaer SL, Cars 0, Buchholz U, Mölstad S, Goettsch W, Veldhuijzen IK,et al.: A European study on the relationship between antimicrobial use and antimicrobial resistance. Emerg Infect Dis. 2002; 8: 278-82.

21. London N, Nijsten R, Mertens P, v d Bogaard A, Stobberingh $\mathrm{E}$ : Effect of antibiotic therapy on the antibiotic resistance of faecal Escherichia coli in patients attending general practitioners. J Antimicrob Chemother. 1994; 34: 239-46.

22. Burns JL: Mechanisms of bacterial resistance. Pediatr Clin North Am. 1995; 42: 497-507.
23. Howard AJ, Magee JT, Fitzgerald KA, Dunstan FD; Welsh Antibiotic Study Group: Factors associated with antibiotic resistance in coliform organisms from community urinary tract infection in Wales. J Antimicrob Chemother. 2001; 47: 305-13.

24. Saffar MJ: Acute respiratory tract infections the main causes for antibiotic prescription in children. Third annual congress of Iranian Society of Pediatric Infectious Diseases, 23-24 Apr 2008, Tehran-Iran, pp. 60-9.

25. Sharifian M, Karimi A, Tabatabaei SR, Anvaripour N: Microbial sensitivity pattern in urinary tract infections in children: a single center experience of 1,177 urine cultures. Jpn J Infect Dis. 2006; 59: 380-2.

26. Matsumoto T, Muratani T: Newer carbapenems for urinary tract infections. Int J Antimicrob Agents. 2004; 24(Suppl 1): S35-8.

27. Ferry S, Burman LG, Holm SE: Clinical and bacteriological effects of therapy of urinary tract infection in primary health care: relation to in vitro sensitivity testing. Scand J Infect Dis. 1988; 20: 535-44.
Correspondence address:
Dr. Mohammed Jafar Saffar
Department of Pediatrics
Bouali-Cina Hospital
Pasdaran Boulevard, Sari
Mazandaran Province, Iran
Fax: +981 51 223-4506
E-mail: saffar@softhome.net 